\title{
On the Minimum Quantum Requirement of Photosynthesis
}

Yuzeir Zeinalov

Institute of Biophysics, Bulgarian Academy of Sciences, Academic G. Bonchev str., bl. 21, Sofia 1113, Bulgaria. E-mail: zeinalov@bio21.bas.bg

Z. Naturforsch. 64c, 673-679 (2009); received May 11/June 29, 2009

An analysis of the shape of photosynthetic light curves is presented and the existence of the initial non-linear part is shown as a consequence of the operation of the non-cooperative (Kok's) mechanism of oxygen evolution or the effect of dark respiration. The effect of nonlinearity on the quantum efficiency (yield) and quantum requirement is reconsidered. The essential conclusions are: 1) The non-linearity of the light curves cannot be compensated using suspensions of algae or chloroplasts with high $(>1.0)$ optical density or absorbance. 2) The values of the maxima of the quantum efficiency curves or the values of the minima of the quantum requirement curves cannot be used for estimation of the exact value of the maximum quantum efficiency and the minimum quantum requirement. The estimation of the maximum quantum efficiency or the minimum quantum requirement should be performed only after extrapolation of the linear part at higher light intensities of the quantum requirement curves to "0" light intensity.

Key words: Photosynthetic Light Curves, Quantum Efficiency or Quantum Yield of Photosynthesis, Quantum Requirement of Photosynthesis

\section{Non-Additiveness in the Action of Light in Photosynthesis of Green Plants}

Studies on the light curves of photosynthesis (the dependence of the intensity of photosynthesis on the light intensity) were carried out already in the second half of the 19th century (see Rabinowitch, 1951). It is generally accepted that, with small exceptions, light curves have an initial linear part at low and middle light intensities after which, at higher light intensities, the curves enter the region of saturation or limitation by the darkdependent reactions of the photosynthetic process and reach the so-called light plateau (light saturation region).

It was shown in some experiments that instead of an initial linear part in some cases a logarithmic dependence was observed, whereas in others an exponential one; but nevertheless these curves are accepted as an exception to the "general rule".

The impressive discovery of Joliot et al. (1969), according to which the first saturating and short (several $\mu \mathrm{s}$ ) flash, applied to 5-10 min darkadapted alga or chloroplast suspension, did not lead to oxygen evolution and the yields from the following flashes were oscillating with a periodicity of four, was interpreted by Kok et al. (1970) with the so-called four-step linear model.
According to Kok's scheme (Kok et al., 1970) the photochemical reactions in the water-splitting system of photosynthesis start after absorption of light quanta and are connected with successive accumulation of positive charges in the respective $\mathrm{S}_{i}$ states, and after reaching the $\mathrm{S}_{4}{ }^{+++}$state one oxygen molecule is evolved and the last state passes into $\mathrm{S}_{0}$. The $\mathrm{S}_{1}, \mathrm{~S}_{2}$, and $\mathrm{S}_{3}$ states can be deactivated by inverse reactions designated by the rate constants $k_{1}, k_{2}$, and $k_{3}$. Usually, $k_{1}$ is 0 and the $S_{1}$ state is stable in the dark, which explains the accumulation of the oxygen evolving-centres in this state after adaptation of the cells or chloroplasts for several minutes to the darkness. It is generally accepted that the photosynthetic oxygen production is realized only by Kok's mechanism and that the photoproducts (or the accumulated positive charge, obtained in the higher oxidized $\mathrm{S}_{2}$ and $\mathrm{S}_{3}$ states) are deactivated in the darkness and the effects of the absorbed photons are lost. An approximate analysis (Zeinalov, 1977) carried out without using electronic computing devices led to the following dependence of the oxygen evolution rate $\left(V_{\mathrm{O}_{2}}\right)$ on the irradiance intensity $\rho=\sigma I$, where $I$ is the irradiance intensity and $\sigma$ is a constant depending on the radiant absorption properties of the photosynthesizing system:

$$
V_{\mathrm{O}_{2}}=\rho \mathrm{S}_{3}=\rho^{2} \mathrm{~S}_{2} /\left(\rho+k_{3}\right),
$$


where $k_{3}$ is the deactivating rate constant of the $\mathrm{S}_{3}$ state.

When the intensity of the excitation light is low, i.e. $\rho<<k_{3}$, then $V_{\mathrm{O}_{2}} \approx \rho^{2} \mathrm{~S}_{2} / k_{3}$. Consequently, under this condition there will be a quadratic dependence of the rate of oxygen evolution on the light intensity. At high values of the light intensity, when $\rho>>k_{3}$, then $V_{\mathrm{O}_{2}} \approx \rho \mathrm{S}_{2}$, i.e. a linear dependence will be at hand.

After the publication of our paper (Zeinalov, 1977) we realized that Myers had drawn the same conclusion in 1974. In 1987 Warner and Berry (using a thermodynamic approach) and in 1990 Milin and Sivash confirmed this conclusion independently from our investigations. Franck et al. (1945) and Diner and Mauzerall (1973) observed also a non-linear dependence under anaerobic conditions. In 2006, Govindjee was kind enough to send me several of his own old publications on Emerson's enhancement phenomenon (Govindjee, 1963; Govindjee and Govindjee, 1965), where the non-linearity of the light curves under anaerobic conditions was also presented.

In Fig. 1 an expanded scheme of Kok's model is presented, including the excited $\mathrm{S}_{i}$ states $\left(\mathrm{S}_{i}^{*}\right)$, the forward dark reactions with the rate constant $K_{\mathrm{d}}$, and the back deactivating reactions designated by $K_{1}, K_{2}$, and $K_{3}$. $K$ is a photochemical rate constant depending on the light intensity.

The following system of differential equations describes the kinetics of the centres in all eight states:

$$
\begin{aligned}
& \mathrm{dS}_{0} / \mathrm{d} t=-K \mathrm{~S}_{0}+K_{1} \mathrm{~S}_{1}+K_{\mathrm{d}} \mathrm{S}_{3}^{*}, \\
& \mathrm{dS}_{0}^{*} / \mathrm{d} t=K \mathrm{~S}_{0}-K_{\mathrm{d}} \mathrm{S}_{0}^{*} \\
& \mathrm{dS}_{1} / \mathrm{d} t=K_{\mathrm{d}} \mathrm{S}_{0}^{*}-\left(K+K_{1}\right) \mathrm{S}_{1}+K_{2} \mathrm{~S}_{2} \\
& \mathrm{dS}_{1}^{*} / \mathrm{d} t=K \mathrm{~S}_{1}-K_{\mathrm{d}} \mathrm{S}_{1}^{*} \\
& \mathrm{dS}_{2} / \mathrm{d} t=K_{\mathrm{d}} \mathrm{S}_{1}^{*}-\left(K+K_{2}\right) \mathrm{S}_{2}+K_{3} \mathrm{~S}_{3}, \\
& \mathrm{dS}_{2}^{*} / \mathrm{d} t=K \mathrm{~S}_{2}-K_{\mathrm{d}} \mathrm{S}_{2}^{*} \\
& \mathrm{dS}_{3} / \mathrm{d} t=K_{\mathrm{d}} \mathrm{S}_{2}^{*}-\left(K+K_{3}\right) \mathrm{S}_{3}, \\
& \mathrm{dS}_{3}^{*} / \mathrm{d} t=K \mathrm{~S}_{3}-K_{\mathrm{d}} \mathrm{S}_{3}^{*} .
\end{aligned}
$$

Under steady-state conditions the concentration (the number) of the centres in all states remains constant and, consequently, the derivatives in the left part of the equations equal zero. Accepting that the total number of the reaction centres in a given suspension is constant (for simplicity normalized to 1 ) one of the states (for instance $S_{0}$ ) could be eliminated and the number of the variables will be reduced to 7 .

According to Fig. 1 the rate of oxygen evolution is determined by the expression $V_{\mathrm{O}_{2}}=K_{\mathrm{d}} \mathrm{S}_{3}^{*}$.

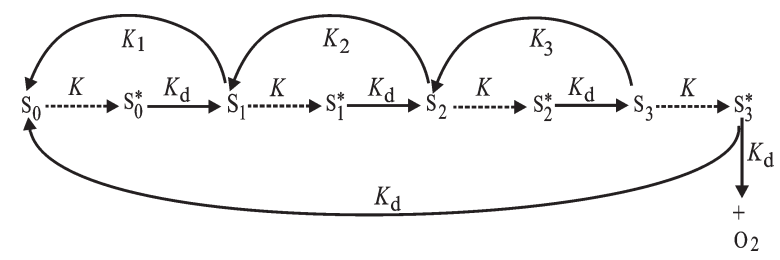

Fig. 1. Scheme of the model of Kok et al. (1970) supplemented by including the back deactivation reactions of the different $\mathrm{S}_{i}$ states with the rate constants $K_{1}, K_{2}$ and $K_{3}$ and forward dark reactions designated by $K_{\mathrm{d}}$, reflecting the capturing of the electron donor. The photochemical steps are shown with dashed arrows and designated by $K$.

Using Cramer's rule the number of the centres in the $S_{3}^{*}$ state could be calculated by the following expression:

$$
\begin{aligned}
& \mathrm{S}_{3}^{*}=K^{4} /\left[K^{4}+\left(K_{\mathrm{d}}+K\right)\left(K_{1}+K\right)\left(K_{2}+K\right)\right. \\
& \left.\left(K_{3}+K\right)\right] .
\end{aligned}
$$

The obtained results are presented in Fig. 2. The non-linearity of the light curve under low light intensity conditions is seen in the inserted figure. The non-linear character is supported by the following experimental data: a) One short flash did not give oxygen yield. b) The yield by the following flashes was a function of the dark interval between the flashes and after a certain optimal dark interval, at which the yield reached its maximum, it gradually decreased. These observations show that: 1 . The photochemical reaction of photosyn-

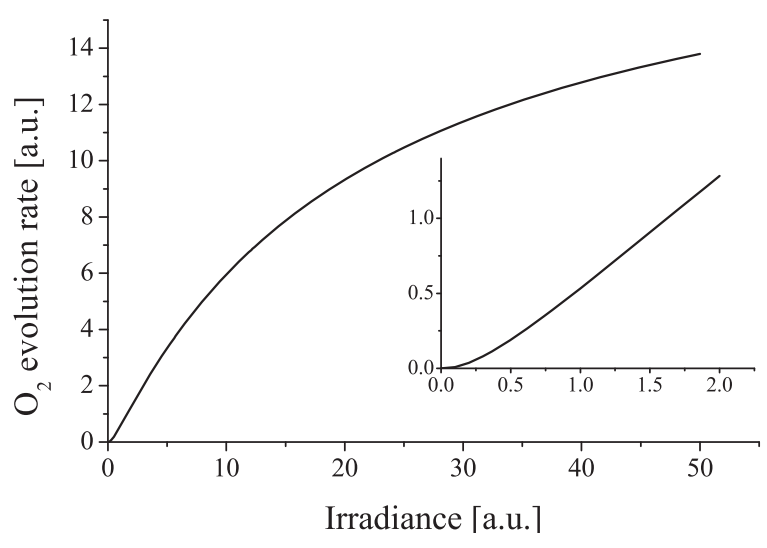

Fig. 2. The calculated light curve of photosynthesis, according to Kok's scheme at $K_{\mathrm{d}}=20 \mathrm{~s}^{-1}, K=50 \mathrm{~s}^{-1}, K_{1}=$ $0 \mathrm{~s}^{-1}, K_{2}=0.05 \mathrm{~s}^{-1}$, and $K_{3}=0.5 \mathrm{~s}^{-1}$. The inset shows the shape of the light curve under low light intensity conditions. 
thetic oxygen production is a multi-quantum (at least four quanta) process. 2 . When the intervals between the flashes increased, the yield of oxygen per given flash decreased, thus indicating that the products obtained as a result of the photochemical reactions (higher oxidized $\mathrm{S}_{i}$ states according to the model of Kok et al.) were unstable and inverse deactivating reactions were taking place.

Consequently, at lower light intensities, when the probability for several successive light quanta going into one and the same reaction centre is very low, the deactivation reactions will dominate in these centres and the quantum yield of photosynthesis will be low. A careful study of the light dependence of photosynthesis shows the non-linear course or S-like shape of the light curves.

In reality, the experimentally obtained light curves show a significantly more pronounced nonlinear dependence, most probably a consequence of dark respiration under anaerobic (or close to anaerobic) conditions in the suspension volumes, investigated with a Warburg manometric method or with Joliot-type polarographic electrode systems.

The results obtained make it possible to express the following statement: On account of the nonlinearity of the light curves of photosynthesis at low light intensities the action of two beams (two light fluxes), even with one and the same wavelength, is not an additive one, i.e., the effect of the two beams operating simultaneously will not be equal to the sum of the effects of the two beams upon their independent action.

This conclusion is illustrated in Fig. 3. Upon the independent action of the two beams with the intensities $I_{1}$ and $I_{2}$, their effects will be $V_{I_{1}}$

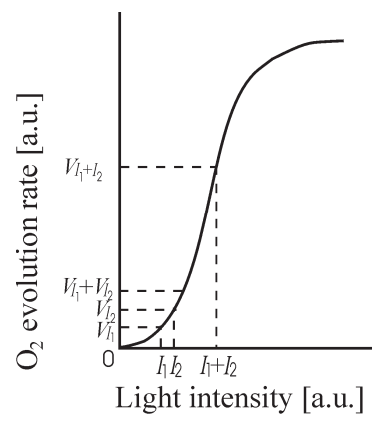

Fig. 3. A graphic explanation of the non-additive action of the light. For details see the text. and $V_{I_{2}}$, respectively. Upon the simultaneous action of the two beams the summarized intensity of the light will be $I_{1}+I_{2}$ with its corresponding intensity of $V_{I_{1}+I_{2}}$ photosynthesis or of oxygen release of which is appreciably higher than the sum of $V_{I_{1}+I_{2}}$. Consequently, we should accept that the effect of light on photosynthesis is nonadditive not only for light beams with different wavelengths (Emerson's enhancement effect) but also for beams with equal wavelengths.

\section{Effect of Suspension Optical Density on the Light Curves of Photosynthesis}

We have shown that there are at least two reasons which could lead to the non-linearity of the photosynthetic light curves under low irradiance conditions. The first one is the deactivation of the higher oxidized $\mathrm{S}_{i}$ states in the widely accepted Kok's model. The second reason is the respiration of the photosynthesizing systems. Despite the fact that we do not accept the statement that in the process of deactivation of the higher oxidized $\mathrm{S}_{3}$ and $S_{2}$ states the obtained oxygen precursors (or positive charges) are lost and thus not included in oxygen evolution, we will accept the theoretically calculated non-linearity of the light curves [obtained by (3), see Fig. 2] for convenience as the minimum non-linearity. As it was explained, the experimentally registered curves are with significantly more pronounced non-linearity.

Let have a layer of a suspension with certain optical density $(A)$ and divide it into $n$ sublayers with equal $(a=A / n)$ optical densities. Suppose that a light beam with intensity $I_{0}$ falls on the upper surface of the suspension. The intensity of this beam decreases gradually because of absorption in the different sublayers, and the light intensity on the sublayer bounds is designated as $I_{1}, I_{2}, I_{3}$ etc., where the index indicates the number of the layer from which the beam comes. Suppose also that the Lambert-Beer law is valid for this suspension, so that the values of $I_{i}$ may be calculated by the following expression:

$$
I_{i}=I_{0} / 10^{i a} .
$$

From this dependence, for the quantity of light energy, absorbed in a given layer for a unit of time, the following expression will be obtained:

$$
\Delta I_{i}=I_{i-1}-I_{i}=I_{0} / 10^{(i-1) a}-I_{0} / 10^{i a},
$$


which presents the differences between the intensities of incoming and outgoing light fluxes in the layer $i$.

In Fig. 4 ten light curves of the individual sublayers of a suspension with 5.0 (Fig. 4A) and 1.0 (Fig. 4B) optical densities calculated using the expressions (3) and (4) are shown. This ratio of optical densities is approximately equal to the absorption properties of a unicellular green alga (like Chlorella and Scenedesmus) suspension at $680 \mathrm{~nm}$ and $700 \mathrm{~nm}$ wavelengths.

The data show that in the high optical density suspension (5.0), when the first sublayer was saturated, the oxygen evolution rates at the 8th, 9th and 10th sublayers were close to zero. On the other hand, the light curves for the different sublayers in the suspension with low absorbance (1.0) (Fig. 4B) were similar and all sublayers participated effectively in the process of oxygen evolution.

Considering the curves presented in Fig. 4A and $\mathrm{E}$ it is not clear how we should select the irradiance intensity at which all light curves of the different sublayers will be in their linear parts. It is seen (Figs. 4B and F) that only under conditions of low absorbance nearly all sublayers and the entire suspension could be approximately situated in the linear parts of their own photosynthetic light curves.

The results presented in Fig. $4 \mathrm{C}$ show also that the saturation or close-to-saturation state of the entire suspension layer light curve is reached under very low light intensities. Under such intensities, the essential part of the suspension sublayers do not participate in the process of photosynthesis. This means that when we compensate for the initial, non-linear part of the entire suspension layer, this does not mean that we compensate for the non-linearity of the light curves of the individual sublayers.

\section{Quantum Efficiency of Photosynthesis with Non-Linear Light Curves}

Using (3), (4) and (5) the calculated quantum efficiency curves for the individual sublayers are presented in Figs. 5A and B. It is evident that all curves exhibit equal amplitude maxima, however,
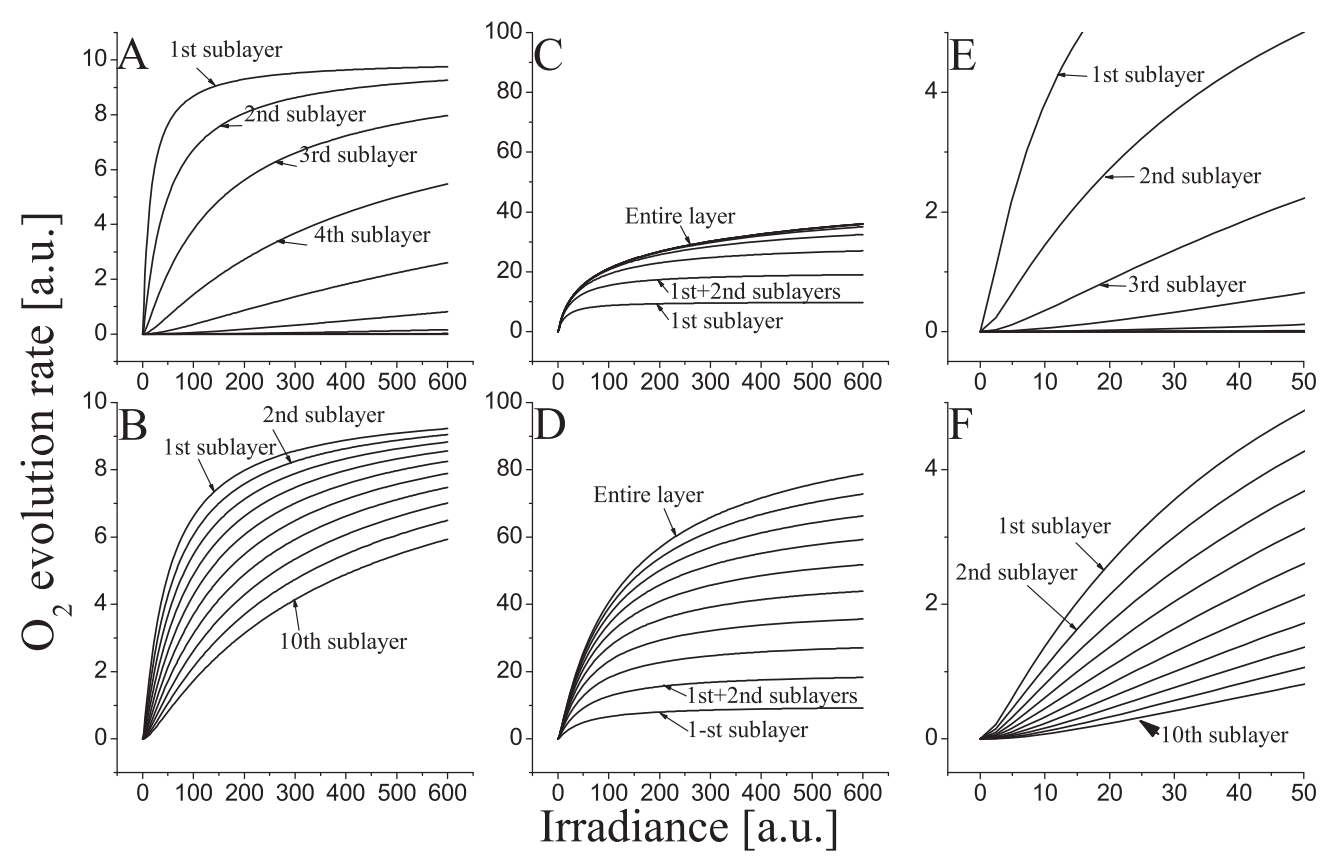

Fig. 4. The individual light curves calculated according to Kok's model for 10 sublayers of a suspension with (A) 5.0 the first row (at $680 \mathrm{~nm}$ ) and (B) 1.0 the second row (at $700 \mathrm{~nm}$ ) optical densities, the calculated light curves for suspensions with different optical densities from (C) 0.5 to 5.0 and (D) from 0.1 to 1.0 , and (E, F) the initial part of the curves presented in the panels A and B, respectively. The rate constants included are the same as in Fig. 2. 
situated in different regions of the light intensity as a consequence of the decreased intensity for the lower situated sublayers. It should be noted that the maxima of the quantum efficiency for the suspension with low optical density (1.0) are situated in a very narrow region of irradiation (Fig. 5B) while those for higher optical density (5.0 - Fig. 5A) are so dispersed that only maxima for the first four or five sublayers are seen under the considered maximal irradiance intensity.

Data presented show that the maximum value of the quantum efficiency for the entire suspension layer in the suspension with higher optical density (5.0) (Fig. 5C) was only about $90 \%$ of the maximum value for the first sublayer. The quantum efficiency of the entire layer represents an average value of the quantum efficiency of different sublayers, which cannot be in the linear part of their individual light curves simultaneously in optically dense suspensions.

In many investigations on the quantum efficiency the reciprocal value $\phi^{-1}$ of the quantum efficiency, the quantum requirement, is used. The explanation of this dependence is presented in Fig. 6 where part a shows the quantum efficiency for the entire layer in dependence of the irradiation of a suspension with 5.0 absorbance, presented in Fig. 5C, and part b reflects the same data for the reciprocal value as quantum requirement.

Without having in mind the consequences arising from the non-linearity of the photosynthetic light curves as well as the suspension optical densities, Govindjee (1999), considering the results of Warburg et al. (1969), used an extrapolation line between the first 3 points (Fig. 6c, arrow A) showing that the minimum quantum requirement, presented in the last paper of Warburg, is higher than 8. However, considering the theoretically obtained results in this work, it is evident that we should use the last two or maximum three points at the linear part of the quantum requirement curve, which will lead to 6 quanta for $1 \mathrm{O}_{2}$ molecule (Fig. 6c, arrow B). As it was said, the linear reduction of the quantum requirement

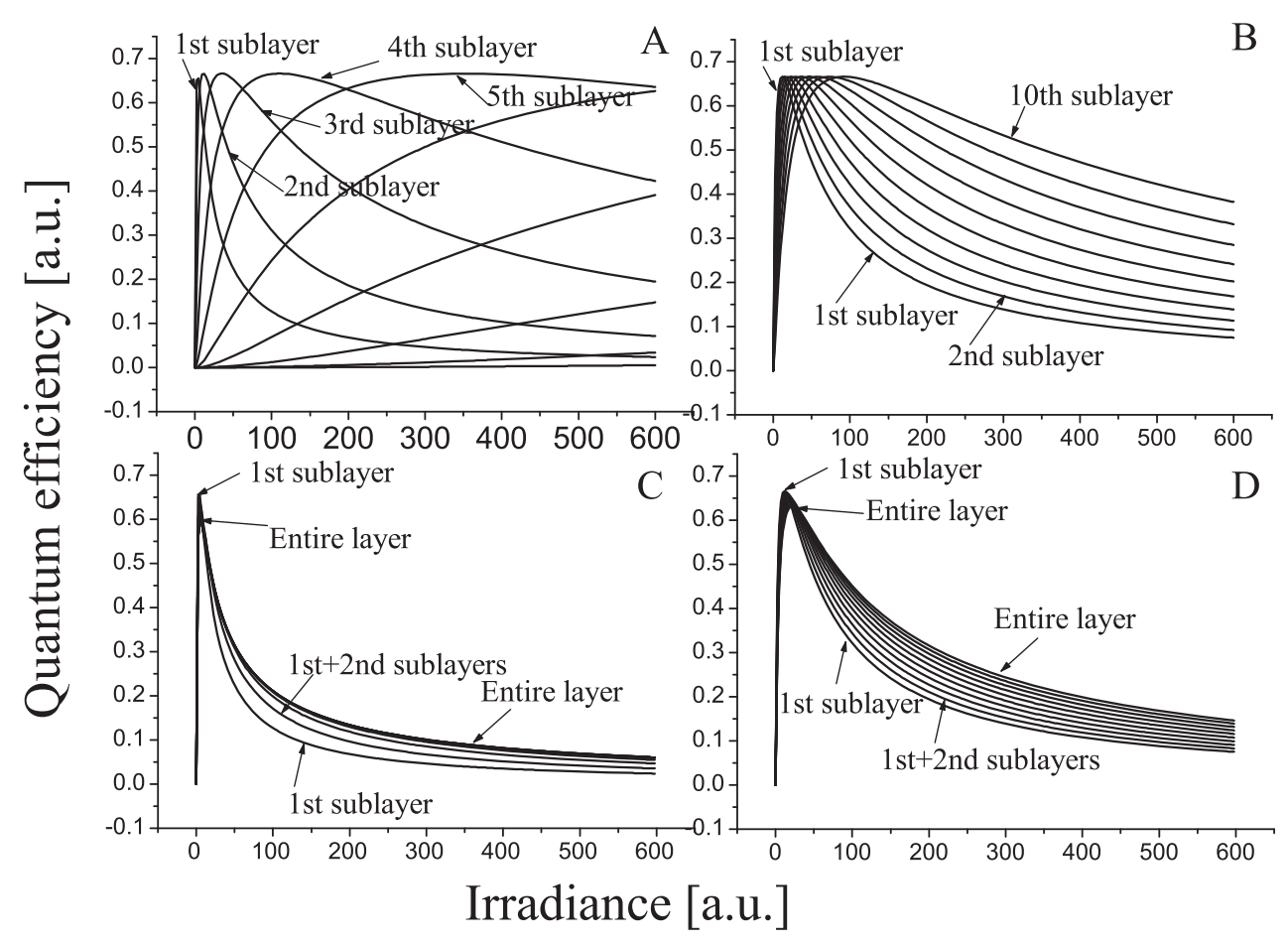

Fig. 5. The quantum efficiency curves for the same sublayers and for suspensions with different optical densities which are shown in Fig. 4. (A, B) The quantum efficiency curves for ten sublayers with 0.5 and 0.1 absorbances, respectively. (C, D) The quantum efficiency curves for suspensions with different absorbance, from 0.5 to 5.0 and from 0.1 to 1.0 , respectively. 

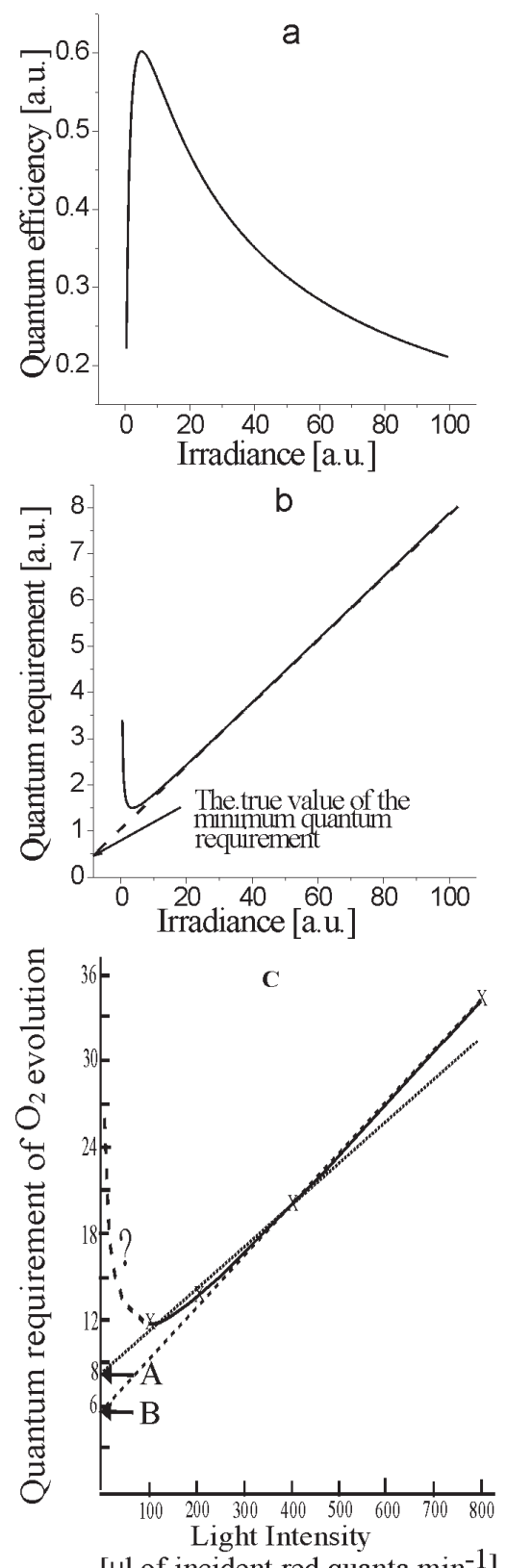

[ $\mu \mathrm{l}$ of incident red quanta $\left.\mathrm{min}^{-1}\right]$ with the decrease of the irradiance intensity is a consequence of the decrease in the number of quanta absorbed in excess that cannot be used effectively. Lowering of the light intensity increases the portion of the effectively used light quanta and decreases the value of the quantum requirement. Since our aim was to determine the value at which every absorbed quantum would be used effectively, we should extend (continue) the extrapolation line to zero light intensity. Only under "zero" light intensity we will be sure that the second quantum will not hit a "Closed R.C." and its energy will not be lost!

In summary we can say that Otto Warburg in his last paper has obtained experimentally 12 quanta for one produced oxygen molecule as a minimum value of quantum requirement. However, having in mind the thickness of the used suspensions, the non-efficient light absorption from the surface sublayers as a consequence of the saturation state, reached in these sublayers under higher intensities, as well as the effect of non-linearity of the light curves under low irradiance conditions the obtained value of the quantum requirement should be reduced below 6, i.e. 5-6 quanta for one oxygen molecule. This correction, i.e. a $40-50 \%$ reduction, should be applied to all early experimental results on the estimation of the minimum quantum requirement!

Fig. 6. (a) Theoretically calculated quantum efficiency and (b) quantum requirement curves for a suspension layer with 5.0 absorbance. (c) Quantum requirement of photosynthesis in Chlorella in dependence on the irradiance. The figure is redrawn from the paper by Warburg et al. (1969) $(1 \mu \mathrm{mol}$ quanta $=22.4 \mu \mathrm{l}$ quanta $=1$ $\mu$ Einstein). The arrow A shows the extrapolation value of the quantum requirement (dotted line) according to Govindjee (1999) and the arrow B shows the same extrapolation value (dashed line) according to the presented analysis. For details see the text. 
Diner B. and Mauzerall D. (1973), Feedback controlling oxygen production in a cross-reaction between two photosystems in photosynthesis. Biochim. Biophys. Acta 305, 329-352.

Franck J., Pringsheim P., and Lad D. (1945), Oxygen production by anaerobic photosynthesis of algae measured by a new micromethod. Arch. Biochem. 7, $103-142$.

Govindjee (1963), Emerson enhancement effect and two light reactions in photosynthesis. In: Photosynthetic Mechanisms in Green Plants (Kok B. and Jagendorf A. T., eds.). National Academy of Sciences - National Research Council, Washington, D.C., publication no. 1145, pp. 318-334.

Govindjee (1999), On the requirement of minimum number of four versus eight quanta of light for the evolution of one molecule of oxygen in photosynthesis: A historical note. Photosynth. Res. 59, 249-254.

Govindjee and Govindjee R. (1965), Two different manifestations of enhancement in the photosynthesis of Porphyridium cruentum in flashing monochromatic light. Photochem. Photobiol. 4, 401-415.

Joliot P., Barbieri G., and Chabaud R. (1969), Un nouveau modele des centre photochimique du systeme II. Photochem. Photobiol. 10, 309-329.
Kok B., Forbush B., and McGloin M. (1970), Co-operation of charges in photosynthetic $\mathrm{O}_{2}$ evolution. I. A linear four step mechanism. Photochem. Photobiol. 11, $457-475$.

Milin A. B. and Sivash A. (1990), Effect Emersona: Novij podhod (The effect of Emerson: A new approach). Fiziologia i biochim. kulturnih rastenii $\mathbf{1}$, 27-31 (in Russian).

Myers J. (1974), Conceptual developments in photosynthesis. Plant Physiol. 54, 420-426.

Rabinowitch E. (1951), Photosynthesis and Related Processes. Vol. 2. Interscience Publishers Inc., New York.

Warburg O., Krippahl G., and Lehman A. (1969), Chlorophyll catalysis and Einstein's law of photochemical equivalence in photosynthesis. Am. J. Bot. 56, 961-971.

Warner J. W. and Berry R. S. (1987), Alternative perspective on photosynthetic yield and enhancement. Proc. Natl. Acad. Sci. USA 84, 4103-4107.

Zeinalov Y. (1977), Non-additiveness in the action of light at the photosynthesis of green plants. Compt. Rend. Acad. Bulg. Sci. 30, 1479-1482. 